\title{
Olfactory neuroblastoma (esthesioneuroblastoma) presenting as ectopic ACTH syndrome: always follow your nose
}

\author{
Karen Decaestecker*, Veerle Wijtvliet*, Peter Coremans and Nike Van Doninck \\ Department of Diabetology-Endocrinology, AZ Nikolaas, Sint-Niklaas, Belgium \\ *(K Decaestecker and $\mathrm{V}$ Wijtvliet contributed equally to this work)
}

Correspondence should be addressed to N Van Doninck

Email

Nike.VanDoninck@

aznikolaas.be

\begin{abstract}
Summary
ACTH-dependent hypercortisolism is caused by an ectopic ACTH syndrome (EAS) in $20 \%$ of cases. We report a rare cause of EAS in a 41-year-old woman, presenting with clinical features of Cushing's syndrome which developed over several months. Biochemical tests revealed hypokalemic metabolic alkalosis and high morning cortisol and ACTH levels. Further testing, including 24-hour urine analysis, late-night saliva and low-dose dexamethasone suppression test, confirmed hypercortisolism. An MRI of the pituitary gland was normal. Inferior petrosal sinus sampling (IPSS) revealed inconsistent results, with a raised basal gradient but no rise after CRH stimulation. Additional PET-CT showed intense metabolic activity in the left nasal vault. Biopsy of this lesion revealed an unsuspected cause of Cushing's syndrome: an olfactory neuroblastoma (ONB) with positive immunostaining for ACTH. Our patient underwent transnasal resection of the tumour mass, followed by adjuvant radiotherapy. Normalisation of cortisol and ACTH levels was seen immediately after surgery. Hydrocortisone substitution was started to prevent withdrawal symptoms. As the hypothalamic-pituitary-axis slowly recovered, daily hydrocortisone doses were tapered and stopped 4 months after surgery. Clinical Cushing's stigmata improved gradually.
\end{abstract}

\section{Learning points:}

- Ectopic ACTH syndrome can originate from tumours outside the thoracoabdominal region, like the sinonasal cavity.

- The diagnostic accuracy of IPSS is not $100 \%$ : both false positives and false negatives may occur and might be due to a sinonasal tumour with ectopic ACTH secretion.

- Olfactory neuroblastoma (syn. esthesioneuroblastoma), named because of its sensory (olfactory) and neuroectodermal origin in the upper nasal cavity, is a rare malignant neoplasm. It should not be confused with neuroblastoma, a tumour of the sympathetic nervous system typically occurring in children.

- If one criticises MRI of the pituitary gland because of ACTH-dependent hypercortisolism, one should take a close look at the sinonasal field as well.

\section{Background}

Ectopic ACTH syndrome (EAS) accounts for $10-18 \%$ of all types of Cushing's syndrome. EAS is most frequently caused by a small-cell lung carcinoma $( \pm 50 \%)$. Olfactory neuroblastoma (ONB) is an extremely rare cause of EAS, of which only 21 cases have been reported. When suspecting an ectopic cause of ACTH hypersecretion, it is important to look beyond the thoracoabdominal region and also consider the sinonasal cavity. This is especially true in 
case of inconsistent IPSS results, due to the possibility of false positives or false negatives.

\section{Case presentation}

A 41-year-old Caucasian woman with prior history of bariatric surgery, IVF-induced pregnancy and post-partum thyroiditis presented with a Cushingoid appearance. Her symptoms developed over 5 months and included rounding of the face, alopecia of the scalp, hirsutism on the upper lip, easy bruisability, amenorrhea and proximal muscle weakness of the lower limbs. She mentioned a pressing headache and slight deterioration of vision. Clinical examination revealed a rounded face, centripetal obesity, proximal muscle atrophy, thinned scalp hair, hyperpigmentation in sun-exposed neck region, ecchymosis and arterial hypertension (Fig. 1).

\section{Investigation}

Multiple laboratory tests were performed, as depicted in Table 1. Blood analysis showed elevated morning cortisol and ACTH levels, hypokalemic metabolic alkalosis and hypernatraemia. Cushing's syndrome was diagnosed with elevated 24-hour urinary cortisol excretion, elevated latenight salivary cortisol and no suppression of morning cortisol after low-dose dexamethasone suppression test. We noted high ACTH and cortisol values at midnight and loss of circadian rhythm as a sign of ACTH dependency (Table 2).

MRI of the pituitary gland showed no pituitary adenoma. Inferior petrosal sinus sampling (IPSS) was performed with use of contrast enhancement to ensure correct catheter placement. Absolute ACTH values during IPSS are displayed in Table 3. Central-to-peripheral ACTH gradient was 2.8: clearly above the cut-off of 2.0 and therefore compatible with Cushing's disease. However, we did not measure the expected rise in ACTH gradient after CRH stimulation, with a central-to-peripheral gradient below 3.0 (Table 3). Additional PET-CT showed intense metabolic activity in the left anterior ethmoidal sinus and left upper nasal turbinate, extending to the middle and lower left nasal cavity (Fig. $2 \mathrm{~A}, \mathrm{~B}$ and C). Reassessment of initial MRI of pituitary gland confirmed the presence of a sinonasal mass.

Anatomopathological investigation of this polypoid lesion showed typical cellular nests of small round blue cells with a high nuclear-to-cytoplasmic ratio, rare nucleoli and a nuclear chromatin pattern typical of neuroendocrine-like tumours (Fig. 3A). Immunohistochemical staining showed positivity for multiple neuro-endocrine
A

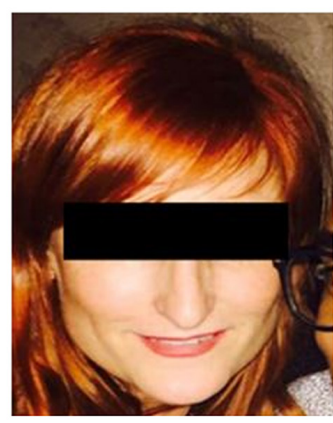

B

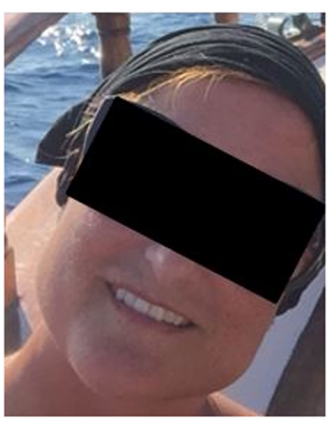

Figure 1

Clinical examination revealed a rounded face (B). Picture $A$ was 2 years ago and was added for comparison. Several months after treatment (C), Cushingoid stigmata clearly improved.

markers, such as CD56, synaptophysin, chromogranin A and INSM1 (Fig. 3B). S100 staining highlighted the support cells surrounding the tumour nests, also known as sustentacular (Schwann) cells (Fig. 3C), needed to differentiate $\mathrm{ONB}$ from melanoma. Staining for pancytokeratine AE1/AE3 marker was negative, ruling out a neuro-endocrine tumour type carcinoid or neuroendocrine carcinoma. Finally, cytoplasm cross-reacted with a mouse ACTH monoclonal antibody, proving ACTH overexpression of the olfactory neuroblastoma (Fig. 3D).

We took a CT scan with thin slices in the coronal plane for better evaluation of the lamina cribrosa. It confirmed a large soft tissue mass in the left nasal vault extending to the lamina cribrosa on one side and to the middle and lower nasal turbinate on the other side. There was no evidence of further protrusion into the anterior cranial fossa nor the orbits (Fig. 2D).

\section{Treatment}

Our patient underwent transnasal endoscopic resection of the tumour mass (Fig. 4). The entire left middle concha was tumourally transformed and removed as far as the lamina cribrosa, which also showed signs of tumoral invasion. The exposed dura mater and nasal 
Table 1 Overview of laboratory results.

\begin{tabular}{|c|c|c|}
\hline & Result & Reference value \\
\hline \multicolumn{3}{|l|}{ Blood } \\
\hline Sodium (mmol/L) & 149 & $135-145$ \\
\hline Potassium (mmol/L) & 2.6 & $3.5-5.1$ \\
\hline Chloride (mmol/L) & 108 & $98-107$ \\
\hline Bicarbonate (mmol/L) & 34 & $21-32$ \\
\hline Urea $(\mathrm{mg} / \mathrm{dL})$ & 26 & $15-39$ \\
\hline Creatinine (mg/dL) & 0.73 & $0.55-1.02$ \\
\hline $\mathrm{CRP}(\mathrm{mg} / \mathrm{L})$ & 13.9 & $<3.0$ \\
\hline Cortisol ( $\mu \mathrm{g} / \mathrm{dL})$ & 27.7 & $10-20$ \\
\hline ACTH $(p g / m L)$ & 66.1 & $10-60$ \\
\hline Cortisol after LDST & 9.1 & $<5$ \\
\hline \multicolumn{3}{|l|}{ 24-hour urine } \\
\hline Volume (mL) & 950 & \\
\hline Cortisol ( $\mu \mathrm{g} / \mathrm{dL})$ & 101.4 & \\
\hline Cortisol ( $\mu \mathrm{g} / 24 \mathrm{~h})$ & 963 & $21-292$ \\
\hline Creatinine (mg/dL) & 71 & $15-278$ \\
\hline Creatinine (mg/24h) & 956 & $800-2000$ \\
\hline Potassium (mmol/24 h) & 17 & $25-125$ \\
\hline \multicolumn{3}{|l|}{ Saliva } \\
\hline Cortisol ( $\mu \mathrm{g} / \mathrm{dL})$ & 0.437 & $<0.1$ \\
\hline
\end{tabular}

ACTH, adrenocorticotropic hormone; CRP, C-reactive protein; LDST, low-dose dexamethasone suppression test.

septum showed no signs of tumour damage. Adjacent sinuses were opened for aspiration of retro-obstructive mucus. Anatomopathological investigation confirmed our previous diagnosis. Totality of resection could not be proven by histology, only macroscopically. Postoperative values of cortisol and ACTH were undetectable (respectively $<5 \mathrm{pg} / \mathrm{mL}$ and $0.8 \mu \mathrm{g} / \mathrm{dL}$ ), suggestive of successful resection.

The tumour was categorised as Kadish stage A (with confinement to the nasal vault) or B (considering the uncertainty of paranasal sinus invasion). We opted for adjuvant radiotherapy (30 sessions of 2 grey; by Volumetric Modulated Arc Therapy (VMAT) technique) given the equivocal Kadish stage, relative high risk of ONB recurrence, our patient's young age and minimal side effects to be expected.

\section{Outcome and follow-up}

Postoperative cortisol and ACTH levels were undetectable. Hydrocortisone substitution was started at a total daily dose of $30 \mathrm{mg}$ distributed in three gifts to prevent withdrawal. As the hypothalamic-pituitary-adrenal axis slowly recovered, daily hydrocortisone dose was tapered and finally stopped 4 months after surgery. Clinical Cushing stigmata slowly improved. Morning serum cortisol and ACTH levels were monitored every 2-3 months. After radiotherapy is finished, control MRI of the sinonasal cavities and nasal endoscopy will be performed.

\section{Discussion \\ Difficulties in diagnosing EAS}

After establishing Cushing's syndrome, several tests are possible to distinguish Cushing's disease from EAS. The more invasive IPSS test is considered to be the most reliable one $(1,2,3,4)$. IPSS is recommended in all patients with ACTH-dependent hypercortisolism with (1) no lesion or a lesion $<6 \mathrm{~mm}$ on MRI and with (2) mixed or negative response on CRH stimulation or dexamethasone suppression tests $(1,5)$. A baseline central-to-peripheral gradient above two or a post-CRH stimulation centralto-peripheral gradient above three indicates pituitary Cushing's ('positive gradient'), whereas the absence of a gradient suggests ectopic Cushing's ('negative gradient'). Initial studies reported a near-perfect sensitivity and specificity of IPSS (3). However, subsequent studies showed that diagnostic accuracy is not always $100 \%$ (1, $2,6)$. False negatives occur in $10-15 \%$ of patients. There are several explanations for such false-negative results. First, sampling errors occur due to technical difficulty of the procedure and/or due to anatomical variations of the petrosal sinus venous system. Correct catheter placement should be verified by using contrast-enhanced fluoroscopy imaging or measuring other pituitary hormones such as prolactin (1). Second, patients must be in a hypercortisolemic state at the time of the IPSS procedure, warranting suppression of endogenic pituitary corticotrophs. Suppression of endogenic ACTH or CRH release ensures that any measurable ACTH in the obtained samples is secreted by the neoplasm (3). This means that any medical treatment to control hypercortisolism must be stopped well in advance $(1,3)$. Thirdly, due to variable

Table 2 Cortisol day curve (blood analysis).

\begin{tabular}{|c|c|c|c|c|c|c|}
\hline & $\begin{array}{c}24 / 10 / 2018 \\
08: 00\end{array}$ & $\begin{array}{c}24 / 10 / 2018 \\
12: 00\end{array}$ & $\begin{array}{c}24 / 10 / 2018 \\
16: 00\end{array}$ & $\begin{array}{c}24 / 10 / 2018 \\
20: 00\end{array}$ & $\begin{array}{c}25 / 10 / 2018 \\
00: 00\end{array}$ & $\begin{array}{c}25 / 10 / 2018 \\
04: 00\end{array}$ \\
\hline Cortisol ( $\mu \mathrm{g} / \mathrm{dL})$ & 17.9 & 14.2 & 30.2 & 15.9 & 22.6 & 15.7 \\
\hline ACTH (pg/mL) & 19.8 & 13.1 & 41.1 & 28.3 & 34.6 & 16.4 \\
\hline
\end{tabular}

ACTH, adrenocorticotropic hormone. 
Table 3 Absolute ACTH values and ACTH IPS:P ratios during IPSS.

\begin{tabular}{|c|c|c|c|}
\hline АСТH (pg/mL) & Basal & 3 min post CRH & 10 min post CRH \\
\hline Peripheral & 11 & 23 & 26 \\
\hline Left IPSS & 27 & 32 & 29 \\
\hline Right IPSS & 31 & 32 & 31 \\
\hline Highest IPS:P ratio & 2.8 & 1.4 & 1.2 \\
\hline
\end{tabular}

ACTH, adrenocorticotropic hormone; IPS:P ratio, inferior petrosal sinus to peripheral ratio; IPSS, inferior petrosal sinus sampling.

ACTH secretion, a positive central-to-peripheral gradient may not be reached at time of IPSS. False-positive results are less frequently seen. One explanation is tumour localisation adjacent to the upstream of pituitary venous drainage, as in our case. Olfactory neuroblastoma and sphenoidal sinus ectopic pituitary adenoma are known for causing such false positive gradients. Another possibility is sampling performed by chance in a eucortisolemic state, that is, by a tumour with intermittent hypercortisolism.
This theory implies rapid recovery of the HPA axis, which is in contrast to the typically slow recovery of the HPA axis seen in daily practice (2).

\section{Olfactory neuroblastoma}

Olfactory neuroblastoma (syn. esthesioneuroblastoma) is a rare malignant neuroectodermal nasal tumour, representing about 3\% of all sinonasal malignancies $(7,8)$. It should not be confused with neuroblastoma, a tumour of the sympathetic nervous system typically occurring in children. The tumour ONB is thought to arise from specialised sensory neuroepithelial (neuroectodermal) olfactory cells, which can be found in the upper nasal cavity. This olfactory epithelium consists of three cell types: basal cells, olfactory neurosensory cells and supporting sustentacular cells (7). All three cell types were histologically identified in our case. Typical clinical features include unilateral nasal congestion,
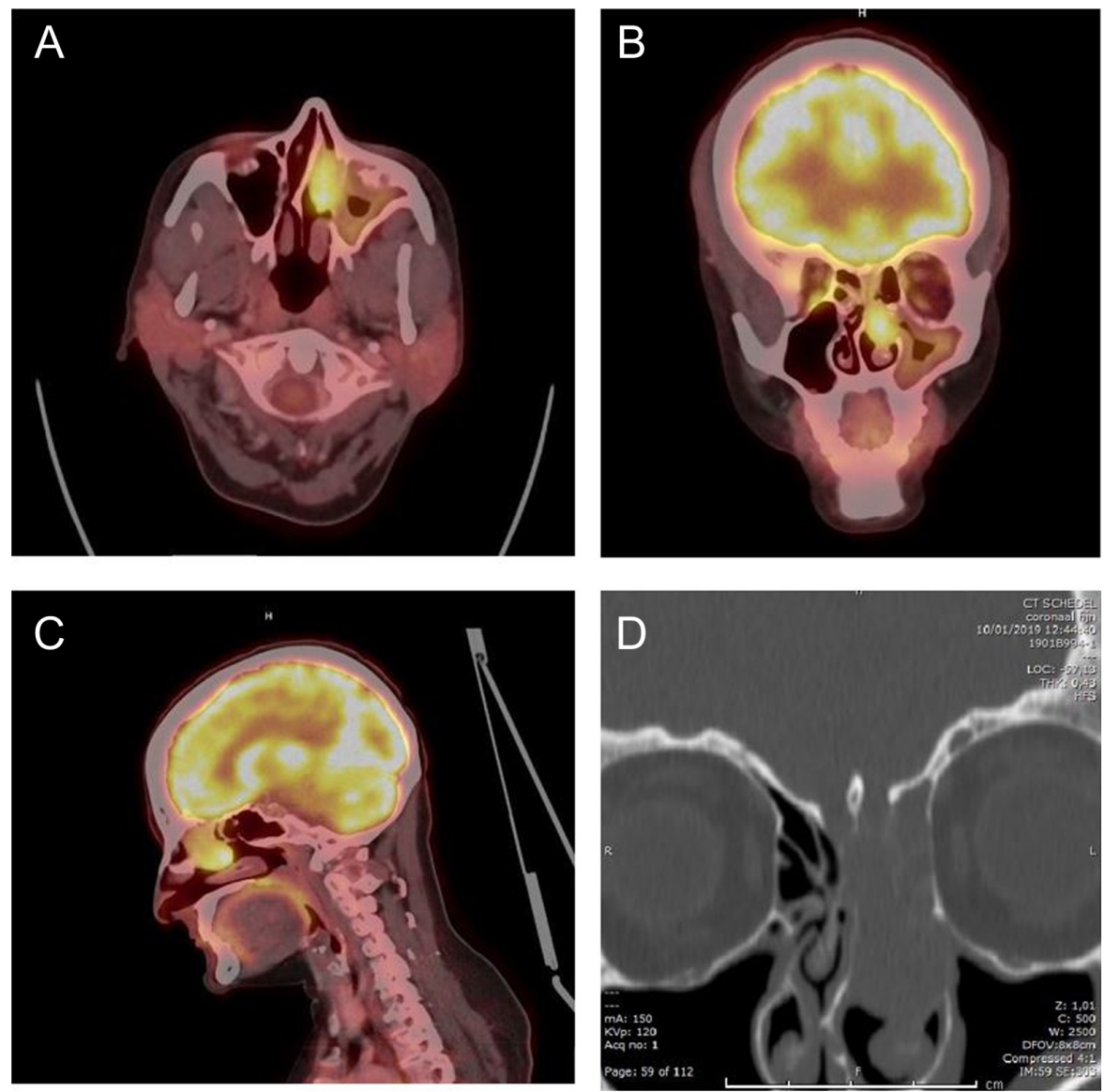

\section{Figure 2}

PET-CT scan showed intense metabolic activity in the left anterior ethmoidal sinus and left upper nasal turbinate, extending to the middle and lower left nasal cavity. Axial (A), coronal (B) and sagittal (C) view are represented. CT scan confirmed a soft tissue mass at the nasal vault extending to the lamina cribrosa. Coronal (D) view is represented. 

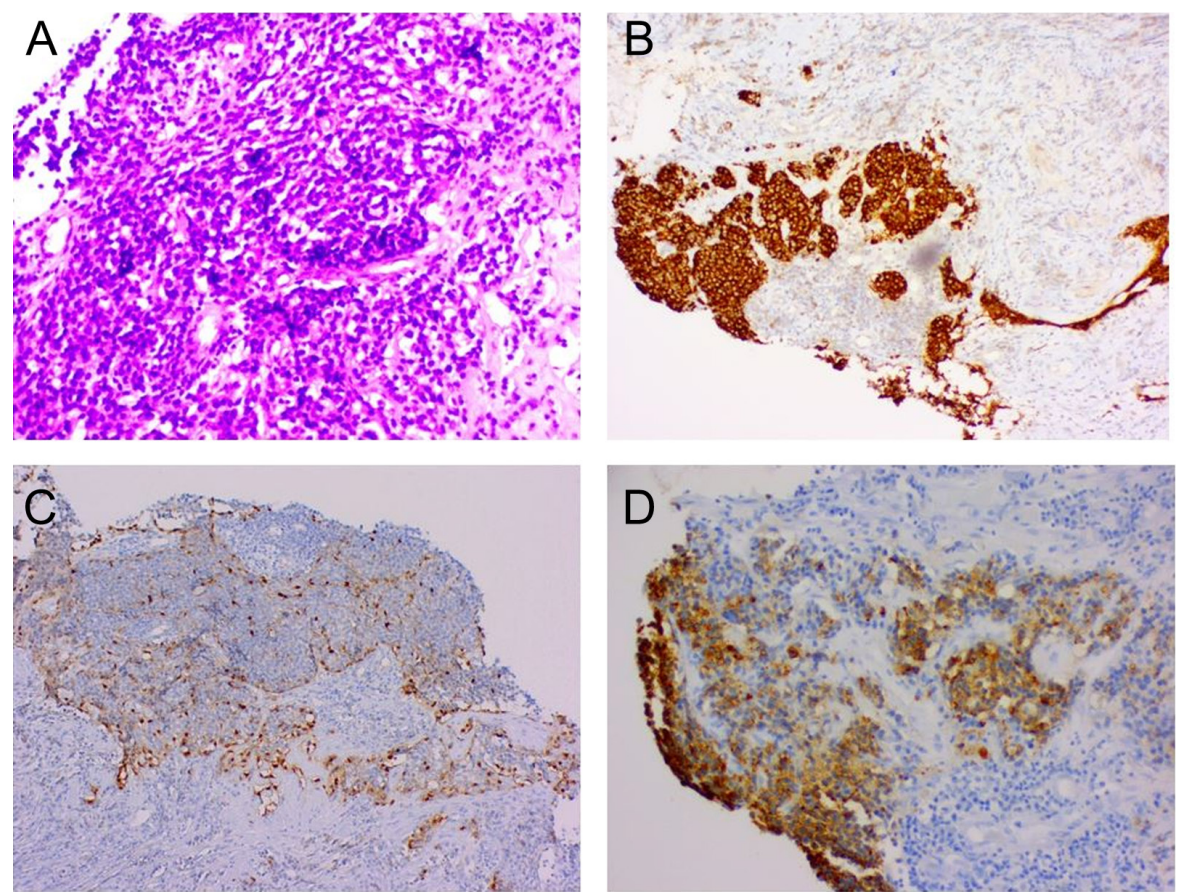

\section{Figure 3}

Anatomopathological investigation showed a pattern typical of neuro-endocrine-like tumours (A). Immunohistochemical staining showed positivity for synaptophysin (B). S100 staining highlighted the support cells surrounding the tumour nests, also known as sustentacular (Schwann) cells (C). Cytoplasm cross-reacted with monoclonal mouse ACTH antibody, proving ACTH overexpression of the olfactory neuroblastoma (D).

recurrent epistaxis, sinusitis, headache and anosmia $(7,8)$. ONB may be associated with endocrine and neurologic paraneoplastic syndromes. SIADH (syndrome of inappropriate $\mathrm{ADH}$ secretion) is most frequently reported (9). ONB presenting with EAS is extremely rare. To our knowledge, only 21 cases have been reported. In 8 of these 21 cases, Cushing's syndrome was the initial presentation of the underlying tumour. For example in $\mathrm{Yu}$ et al. a 55-year-old male presented with muscle weakness, truncal obesity and hypokalemic metabolic alkalosis (10). In Familiar et al. a 31-year-old male presented with persistent hypertension since 2 years and a Cushingoid phenotype (11). A more rapid development of symptoms, hypokalaemia and metabolic alkalosis tend to be typical features of EAS. Of the 21 patients with ONB and EAS, hypokalaemia was mentioned in 14 cases (67\%) and metabolic alkalosis in 9 cases $(43 \%)(9,10,11,12)$.

Multiple staging systems were proposed, but the Kadish staging system is most commonly used. Patients are categorised as (A) tumour limited to the nasal cavity only, (B) involvement of the paranasal sinuses, (C) extension beyond the nose and paranasal sinuses and (D) presence of cervical lymph nodes or distant metastases at time of diagnosis $(7,8,9)$.

Given the rarity of ONB there is no true standard care for treatment, but the mainstay of treatment is surgery (8).
Transnasal endoscopic resection is minimally invasive and is preferred over the traditional, more invasive, open craniotomy with facial incisions. More advanced tumour stages can be a relative contraindication for transnasal approach (8). Adjuvant radiotherapy is administered to minimise the risk of local recurrence, especially in well to moderately differentiated tumours. Chemotherapy is used in poorly differentiated, advanced, unresectable, disseminated or recurrent disease $(7,8)$. There is no standard chemotherapy regimen, but platinum-based regimens combined with etoposide are most commonly used (8).

Reported 5-year survival rates range from 57 to $93 \%(7,8)$. Prognosis of ONB depends on age at diagnosis, Kadish stage, lymph node status and treatment modality (13). Improved survival was observed with multimodality treatment (both surgery and radiotherapy, median 5-year survival rate of $65 \%$ ) as opposed to radiotherapy alone (median 5-year survival rate of $45 \%)(P<0.002)(13)$. In spite of high survival rates, recurrence is very common: about $30 \%$ (range 15-70\%) of patients develop recurrent disease after initial management (7). Recurrence is mostly locoregional, but up to $25 \%$ of patients develop cervical lymph node metastasis and up to $10 \%$ distant metastasis, typically in lungs and bones $(7,8)$. 


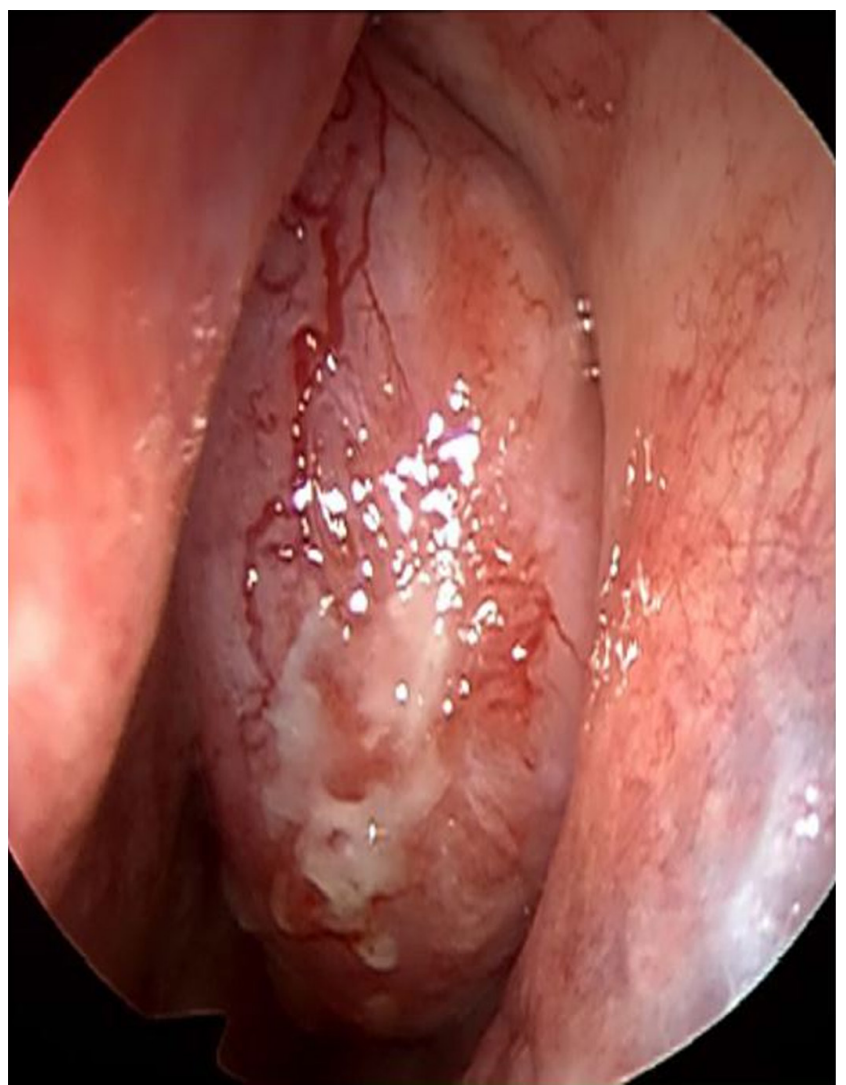

Figure 4

Endoscopic visualisation of the tumour mass in the left nasal cavity. The entire left middle concha was tumourally transformed.

\section{Conclusion}

Olfactory neuroblastoma is an extremely rare cause of EAS. Inconsistent IPSS results might be caused by an ACTH-producing tumour in the sinonasal region, due to its anatomical proximity to the venous drainage of the pituitary gland. In case of EAS, we advise to look beyond the thoracoabdominal region and also consider the sinonasal cavity.

\section{Declaration of interest}

The authors declare that there is no conflict of interest that could be perceived as prejudicing the impartiality of the research reported.

\section{Funding}

This research did not receive any specific grant from any funding agency in the public, commercial or not-for-profit sector.

\section{Patient consent}

Written informed consent was obtained from the patient for publication of this case report and accompanying images.

\section{Author contribution statement}

$\mathrm{KD}$ and $\mathrm{VW}$ both equally contributed to gathering clinical data, writing this article and conducting literature research. N V D and PC managed the case and reviewed the manuscript.

\section{References}

1 Losa M, Allora A, Panni P, Righi C \& Mortini P. Bilateral inferior petrosal sinus sampling in adrenocorticotropin-dependent hypercortisolism: always, never, or sometimes? Journal of Endocrinological Investigation 201942 997-1000. (https://doi.org/10.1007/s40618-019-1006-5)

2 Pecori Giraldi F, Cavallo LM, Tortora F, Pivonello R, Colao A, Cappabianca P, Mantero F \& Altogether to Beat Cushing's Syndrome Group. The role of inferior petrosal sinus sampling in ACTHdependent Cushing's syndrome: review and joint opinion statement by members of the Italian Society for Endocrinology, Italian Society for Neurosurgery, and Italian Society for Neuroradiology. Neurosurgical Focus 201538 E5. (https://doi.org/10.3171/2014.11.FOCUS14766)

3 Utz A \& Biller BM. The role of bilateral inferior petrosal sinus sampling in the diagnosis of Cushing's syndrome. Arquivos Brasileiros de Endocrinologia e Metabologia 200751 1329-1338. (https://doi. org/10.1590/s0004-27302007000800019)

4 Ilias I, Torpy DJ, Pacak K, Mullen N, Wesley RA \& Nieman LK. Cushing's syndrome due to ectopic corticotropin secretion: twenty years' experience at the National Institutes of Health. The Journal of Clinical Endocrinology and Metabolism 200590 4955-4962. (https:// doi.org/10.1210/jc.2004-2527)

5 Nieman LK, Biller BM, Findling JW, Murad MH, Newell-Price J, Savage MO, Tabarin A \& Endocrine Society. Treatment of Cushing's syndrome: an Endocrine Society clinical practice guideline. The Journal of Clinical Endocrinology and Metabolism 2015100 2807-2831. (https://doi.org/10.1210/jc.2015-1818)

6 Zampetti B, Grossrubatscher E, Dalino Ciaramella P, Boccardi E \& Loli P. Bilateral inferior petrosal sinus sampling. Endocrine Connections 20165 R12-R25. (https://doi.org/10.1530/EC-16-0029)

7 Thompson LD. Olfactory neuroblastoma. Head and Neck Pathology 20093 252-259. (https://doi.org/10.1007/s12105-009-0125-2)

8 Abdelmeguid AS. Olfactory neuroblastoma. Current Oncology Reports 201820 7. (https://doi.org/10.1007/s11912-018-0661-6)

9 Kunc M, Gabrych A, Czapiewski P \& Sworczak K. Paraneoplastic syndromes in olfactory neuroblastoma. Contemporary Oncology 2015 19 6-16. (https://doi.org/10.5114/wo.2015.46283)

10 Yu K, Roncaroli F, Kearney T, Ewins D, Beeharry D, Naylor T, Ray D, Bhalla R \& Gnanalingham K. Ectopic Cushing's syndrome secondary to olfactory neuroblastoma. Acta Neurochirurgica $20181601023-$ 1026. (https://doi.org/10.1007/s00701-017-3447-y)

11 Familiar C \& Azcutia A. Adrenocorticotropic hormone-dependent Cushing syndrome caused by an olfactory neuroblastoma. Clinical Medicine Insights. Endocrinology and Diabetes 201912 1179551419825832. (https://doi.org/10.1177/1179551419825832)

12 Kadoya M, Kurajoh M, Miyoshi A, Shoji T, Terada T, Nakamoto Y, Tsukamoto Y, Moriwaki Y, Hirota S \& Koyama H. Ectopic adrenocorticotropic hormone syndrome associated with olfactory neuroblastoma: acquirement of adrenocorticotropic hormone expression during disease course as shown by serial immunohistochemistry examinations. The Journal of International Medical Research 201846 4760-4768. (https://doi. org $/ 10.1177 / 0300060517754026)$

13 Jethanamest D, Morris LG, Sikora AG \& Kutler DI. Esthesioneuroblastoma: a population-based analysis of survival and prognostic factors. Archives of otolaryngology. Head \& Neck Surgery 2007133 276-280. (https://doi.org/10.1001/archotol.133.3.276)

Received in final form 4 September 2019

Accepted 25 September 2019 